\title{
Mobile Programming in Windows Phone and Android Applications and the differences between them
}

\author{
Dr. Abdualmajed Ahmed GhalebAl-Khulaidi (Ph.D) \\ Assistant Professor at Software Engineering at Sana'a University, Computer science section, college of Education, Arts and Sciences,Marib \\ Branch, Yemen
}

\begin{abstract}
This paper intends to discuss the uses,applications and android applications and Iphoneprogramming of in operation system windows phone. It will also explain Windows Phone Platform to Android Application Developers, the developer tools and Windows Phone archticture. We will deal with Windows Phone Silverlight applications in navigation between pages.In fact, Windows Phone applications almost always consist of several pages, and all phones on Windows have a return button . In addition, there would be a explantion for Controls and the Application Interface in Windows Phone and a comparison of Android and Windows Phone Tools. This article will constitute the starting point for all future researchers in mobile programming, android applications and programmers using C\# language in the field of programming Iphone in operation system windows phone.As has been known, nowadays, smart phone specialists have a strong trend towards mobile programming and android applications, a fact which supports the topic of this present article and makes it a reality.
\end{abstract}

Keywords: Android ,windows Phone, IPhone, application, system

\section{Introduction}

Windows Phone presents an exciting new opportunity for developers to build and monetize mobile applications.It represents a major new investment into mobile computing by Microsoft, and in many ways is a break from the past[1,2,3,4,5].

Windows Phone 7 was first releasedby the Windows Phone mobile for clients. The operating system was released worldwide on October 21, 2010,and in the United States on November 8,2010. It received multiple large updates,the last of which being Windows Phone 7.8, was released in January 2013. Microsoft stopped its support to Windows Phone 7 on October 14, 2014.

Windows Phone initially supports twenty-five languages, with applications available throughout Windows Phone storesor providers in 35 countries and regions.

\section{Windows Phone Platform to Android Application Developers}

Since the release of Windows Phone, developers from around the world have started developing applications for this platform. Windows Phone devices are made available at a number of manufacturers for end-users around the world. Tens of thousands of applications are already available in the Windows phone marketplace. For Windows Phone, Microsoft went back to the drawing board to figure out what type of phone users really want, and built a phone from the scratch . The operating system, the user experience, and the application development platform have all been engineered with users in mind. The revenue opportunities in the Windows Phone marketplace, accompanied by a great set of development tools, make Windows Phone a very attractive platform for developers to build their own applications and games[6,7].

\section{The Developer Tools in Windows Phone}

Microsoft has released a great set of tools for Windows Phone application development. The toolset includes:

- An IDE (for developers): Visual Studio Express for Windows Phone

- A user interface design tool (for designers): Express Blend for Windows Phone

- Silverlight for Windows Phone and XNA Game Studio for Windows Phone

- Windows Phone Emulator to test and debug applications The tools are designed to let you develop end-user applications, business applications, and games.

\section{Windows Phone Architecture}

Like Android operating system, Windows Phone is designed to run on multiple phone sets. To provide a consistent user experience and features that you can rely on, Windows Phone defines a minimum set of hardware specifications that all phones must meet. These include[8,9,10]:

a) ARM7 CPU

b) A DirectX capable GPU

c) Camera

d) Multi-touch capacitive display

e) Standard sensors include:

- A-GPS

- Accelerometer

- Compass

- Proximity and light sensors

There are three standard buttons on the phone: Back, Start, and Search. As has been elaborated below , these buttons provide an easy and natural navigation model for the user.

In Windows Phone, Microsoft provides most of the device driver code. A device manufacturer need only to write a very 


\title{
International Journal of Science and Research (IJSR) \\ ISSN (Online): 2319-7064 \\ Index Copernicus Value (2013): 6.14 | Impact Factor (2015): 6.391
}

small code specific to their device. This improves the consistency and quality across various devices. Windows Phone takes advantage of hardware acceleration through encapsulation layers, such as DirectX. WP7 utilizes a layered architecture which is described in the diagram below.

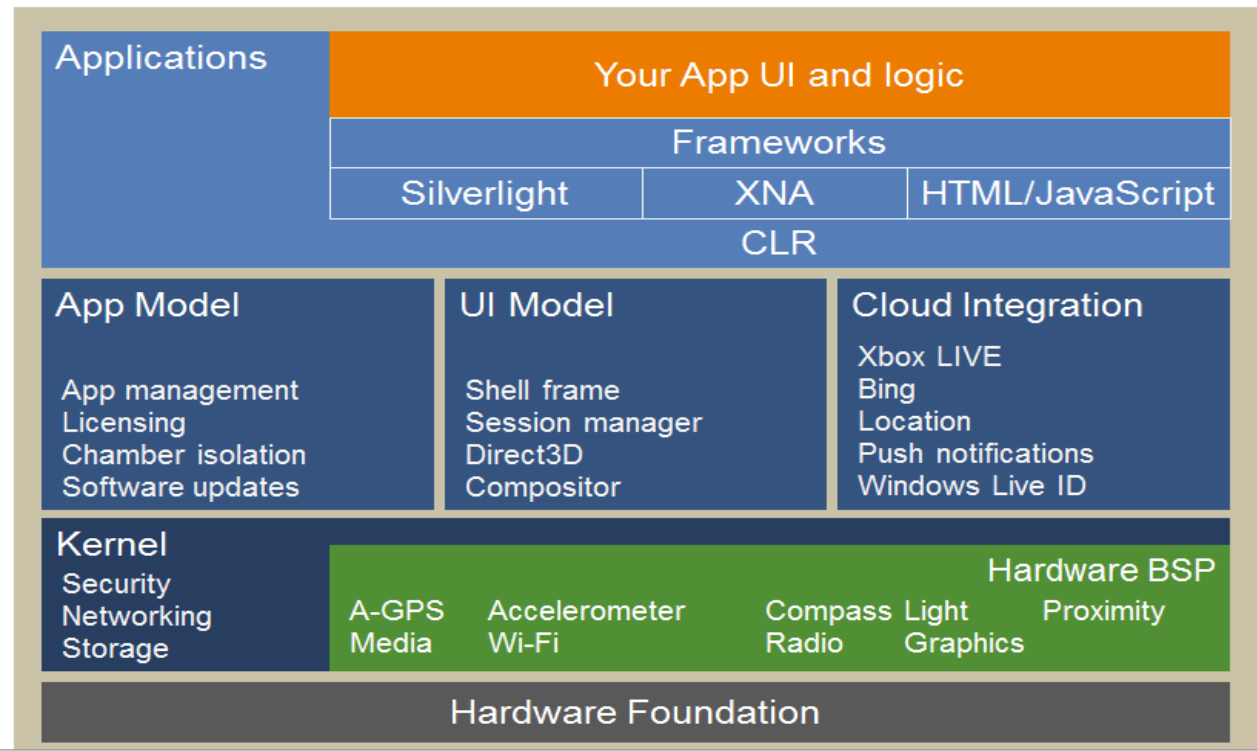

Figure 1: Windows Phone architecture

\begin{abstract}
Windows Phone 7 Silverlight Applications in Navigation among Pages

This point is important because of two reasons. The first is that Windows Phone 7 applications almost consist of many pages, while the second, all phones on Windows 7 have a button "to return." This allows the user to easily return, to the previous page. Navigating among the pages of the phone reminds us of Web applications. When working with the
\end{abstract}

the user's point of view the differences are scant. Windows 7 consists of a single page at least, while Silverlight traditional applications consist of at least one user to control the inside frame (frame) to navigate pages [1].

Windows Phone Portrait Page is for adding a new page in the application; click the Project menu at the top and then add a new item in the dialog box; click on

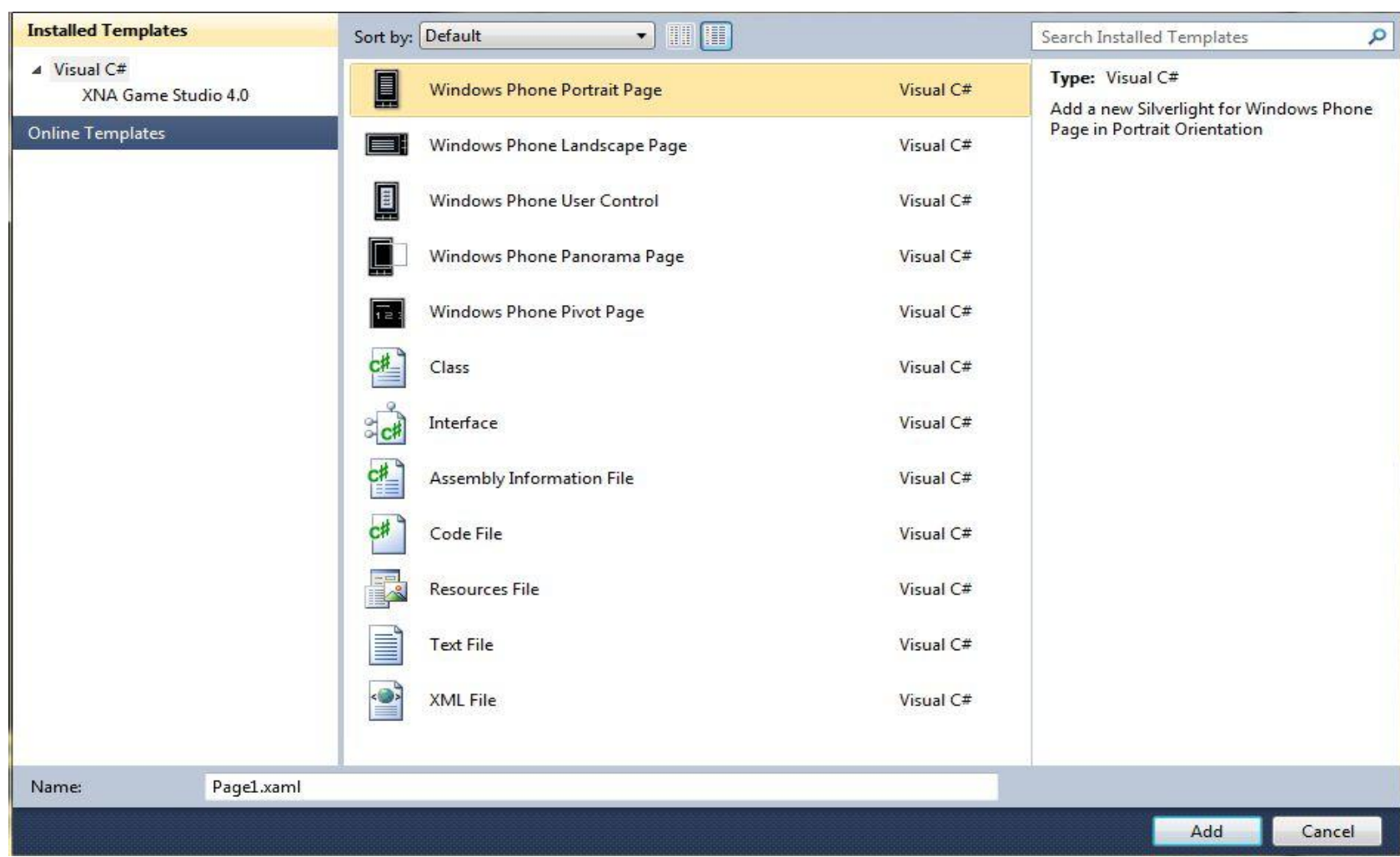

Figure 2: "Application" add a new page in the form 


\section{International Journal of Science and Research (IJSR) \\ ISSN (Online): 2319-7064 \\ Index Copernicus Value (2013): 6.14 | Impact Factor (2015): 6.391}

Responsible to fill out pages. Add three pages and change the title of each page so that the user is defined in any page that supports multi-touch input, and there are usually located three buttons below the screen. Name these buttons in order from left to right:

- Return (Return): programs use this button to move, just like a lot of the back buttons in your web browser. When used with Home.

- Start (Start): This button allows the user to move the phone splash screen.

- Find (search):an operating system uses this key to allow you start your search.

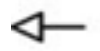

Figure 3: Toolbar buttons

Pixels. $320 * 480$ as in the future will use a screen pixel ize $800 *$ preliminary versions of Windows 7 that the screen size is 480
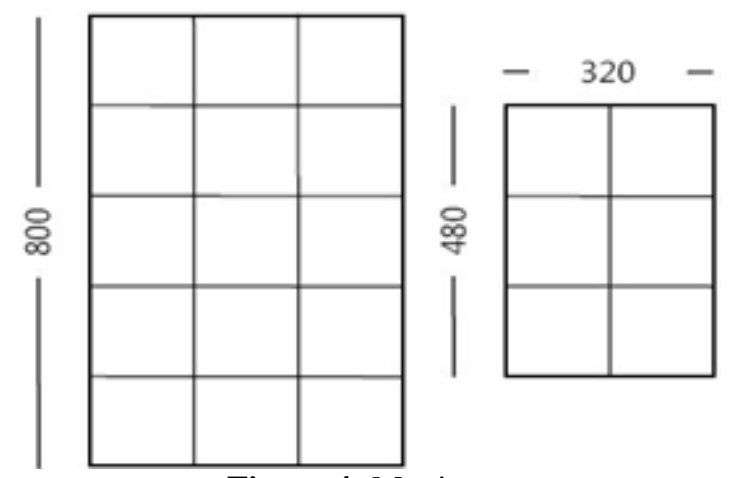

Figure 4: Monitors

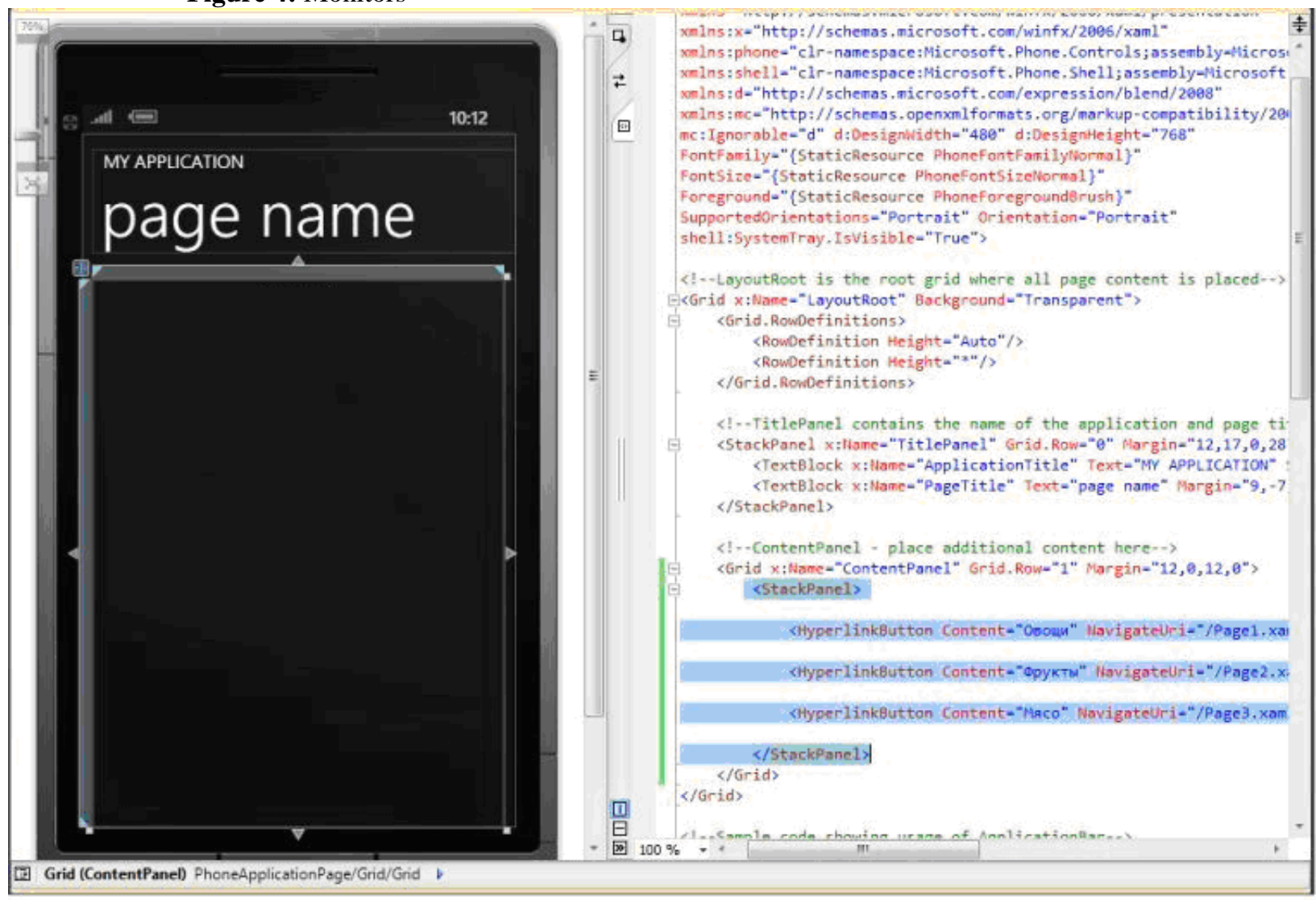

Figure 5: Add links to other pages

Volume 5 Issue 4, April 2016 www.ijsr.net 


\section{International Journal of Science and Research (IJSR) \\ ISSN (Online): 2319-7064 \\ Index Copernicus Value (2013): 6.14 | Impact Factor (2015): 6.391}

. C \# can move on to other pages by writing some code in the "HyperlinkButton" instead of using the control

$<$ Button Content="hello " /> Button add links on the home page button

private void Button_Click (object sender, RoutedEventArgs e)

NavigationService.Navigate (new Uri ("/ Page4.xaml",

UriKind.Relative));

By roads that make us control on movement:

NavigationService kind of class here we use

GoBack1-

GoForward2-

Navigate3-

Refresh4-

StopLoading5-

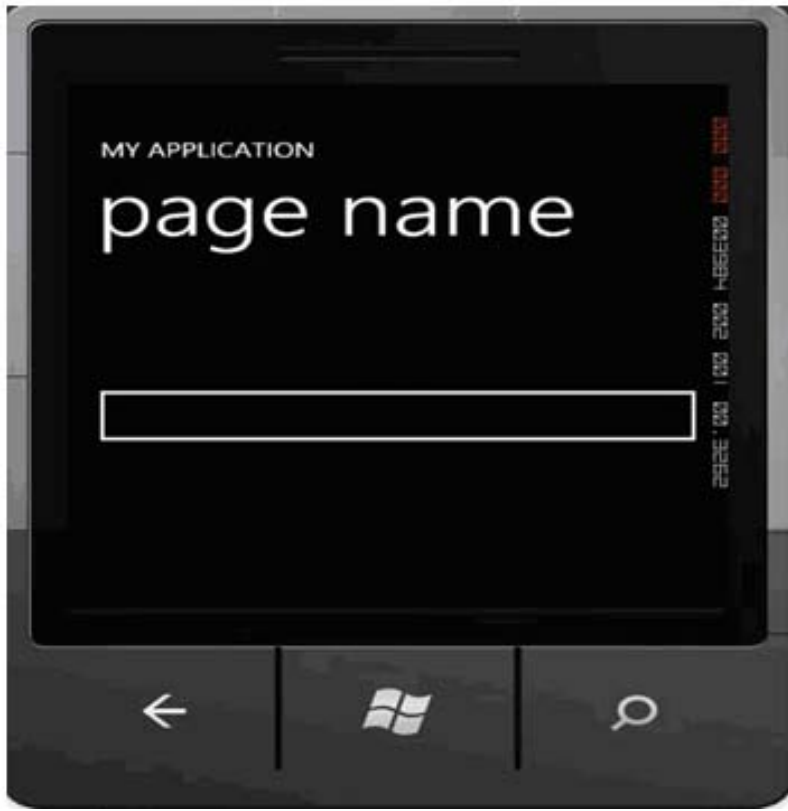

Figure 6: application for iPhone

File-> new-> project->choose XNA Game Studio 4.0. In Visual C\# choose Windows Phone Game (4.0)>name_project.

namespace XnaHelloPhone

\{

public class Game1: Microsoft.Xna.Framework.Gamer

\{

GraphicsDeviceManager graphics;

SpriteBatchspriteBatch;

string text = "Hello, World!";

SpriteFont segoe14;

Vector2 textPosition;

public Game1 ()

\{

graphics = new GraphicsDeviceManager (this);

Content.RootDirectory = "Content";

TargetElapsedTime = TimeSpan.FromTicks (333333);

\}

protected override void Initialize ()

\{

/ / TODO: Add your initialization logic here

base.Initialize ();
\}

protected override void LoadContent ()

\{

spriteBatch = new SpriteBatch (GraphicsDevice);

segoe14 = this.Content.Load $<$ SpriteFont $>$ ("Segoe14");

Vector2 textSize = segoe14.MeasureString (text);

Viewport viewport = this.GraphicsDevice. Viewport;

textPosition $=$ new Vector2 ((viewport.Width - textSize.X) $/$

2, (viewport.Height - textSize.Y) / 2);

\}

protected override void Update (GameTimegameTime) \{

if (GamePad.GetState (PlayerIndex.One). Buttons.Back == ButtonState.Pressed)

this.Exit ();

base.Update (gameTime);

\}

protected override void Draw (GameTimegameTime) \{

GraphicsDevice.Clear (Color.CornflowerBlue);

base.Draw (gameTime);

\}

protected override void Draw (GameTimegameTime)

\{

GraphicsDevice.Clear (Color.Navy);

spriteBatch.Begin ();

spriteBatch.DrawString (segoe14, text, textPosition,

Color.White);

spriteBatch.End ();

base.Draw (gameTime);

\}

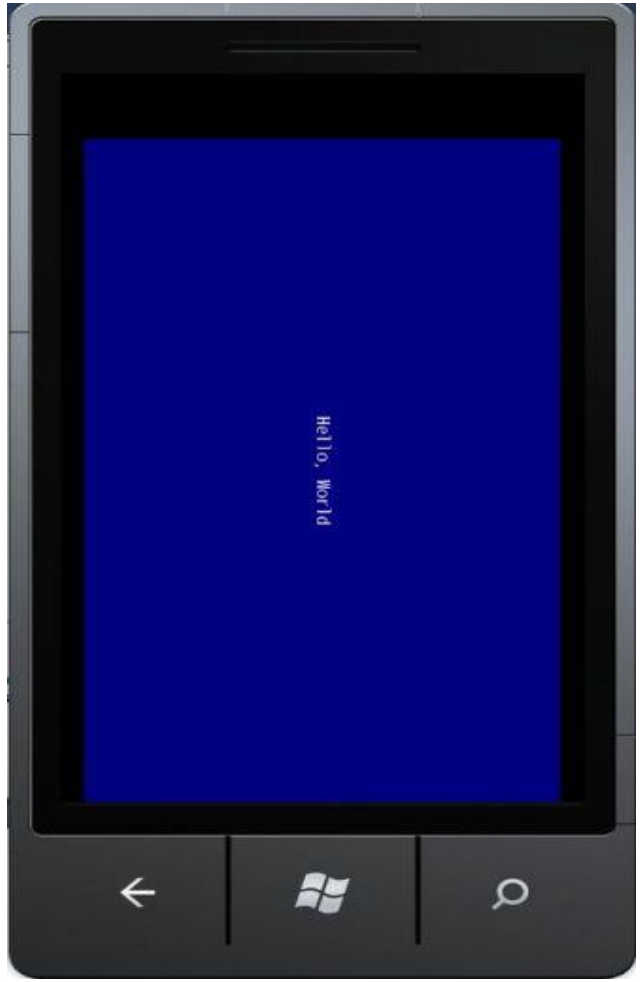

Figure 7: The final application 


\section{International Journal of Science and Research (IJSR) \\ ISSN (Online): 2319-7064}

Index Copernicus Value (2013): 6.14 | Impact Factor (2015): 6.391

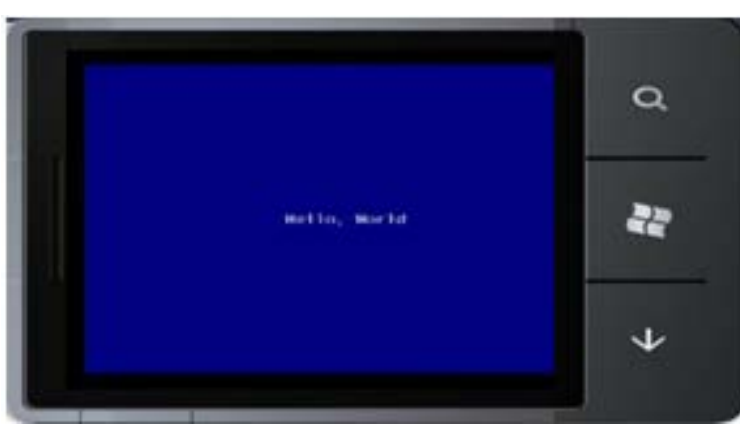

Figure 8: The final application

\section{Controls and the Application Interface in Windows Phone}

The windows Phone development tools and SDK include a rich collection of Silverlight controls that are designed specifically for usability and aesthetics. While you can create your own controls, it is best to use the standard controls wherever possible. These standard controls respond to theme changes and provide a consistent user interface. The following table shows the mapping between Android controls and corresponding windows phone 7 Silverlight controls.

\begin{tabular}{|l|l|}
\hline Android control & Windows Phone control \\
\hline Border & Border \\
\hline ButtonView & Button \\
\hline AbsoluteLayout & Canvas \\
\hline CheckBox & CheckBox \\
\hline GridView & Grid \\
\hline & HyperlinkButton \\
\hline
\end{tabular}

\begin{tabular}{|l|l|}
\hline ImageView & $\underline{\text { Image }}$ \\
\hline ListView & ListBox \\
\hline MediaController & MediaElement \\
\hline & MultiScaleImage \\
\hline ViewGroup & $\underline{\text { Panel }}$ \\
\hline EditText & PasswordBox \\
\hline ProgressBar & ProgressBar \\
\hline RadioButton, RadioGroup & $\underline{\text { RadioButton }}$ \\
\hline ScrollView & $\underline{\text { ScrollViewer }}$ \\
\hline SeekBar & Slider \\
\hline LinearLayout & StackPanel \\
\hline EditText & TextBlock \\
\hline EditText & $\underline{\text { TextBox }}$ \\
\hline MapView & Map \\
\hline WebView & WebBrowser \\
\hline & Panorama \\
\hline & Pivot \\
\hline TimePicker & Timepicker \\
\hline DatePicker & Datepicker \\
\hline ExpandableListView & \\
\hline Gallery & \\
\hline ImageSwitcher & \\
\hline Spinner & \\
\hline TableLayout & Grid Layout \\
\hline TextSwitcher & \\
\hline ViewFlipper & \\
\hline ZoomControl & \\
\hline TabHost & \\
\hline SlidingDrawer & \\
\hline RatingBar & ToggleSwitch* \\
\hline Toggle button & \\
\hline
\end{tabular}

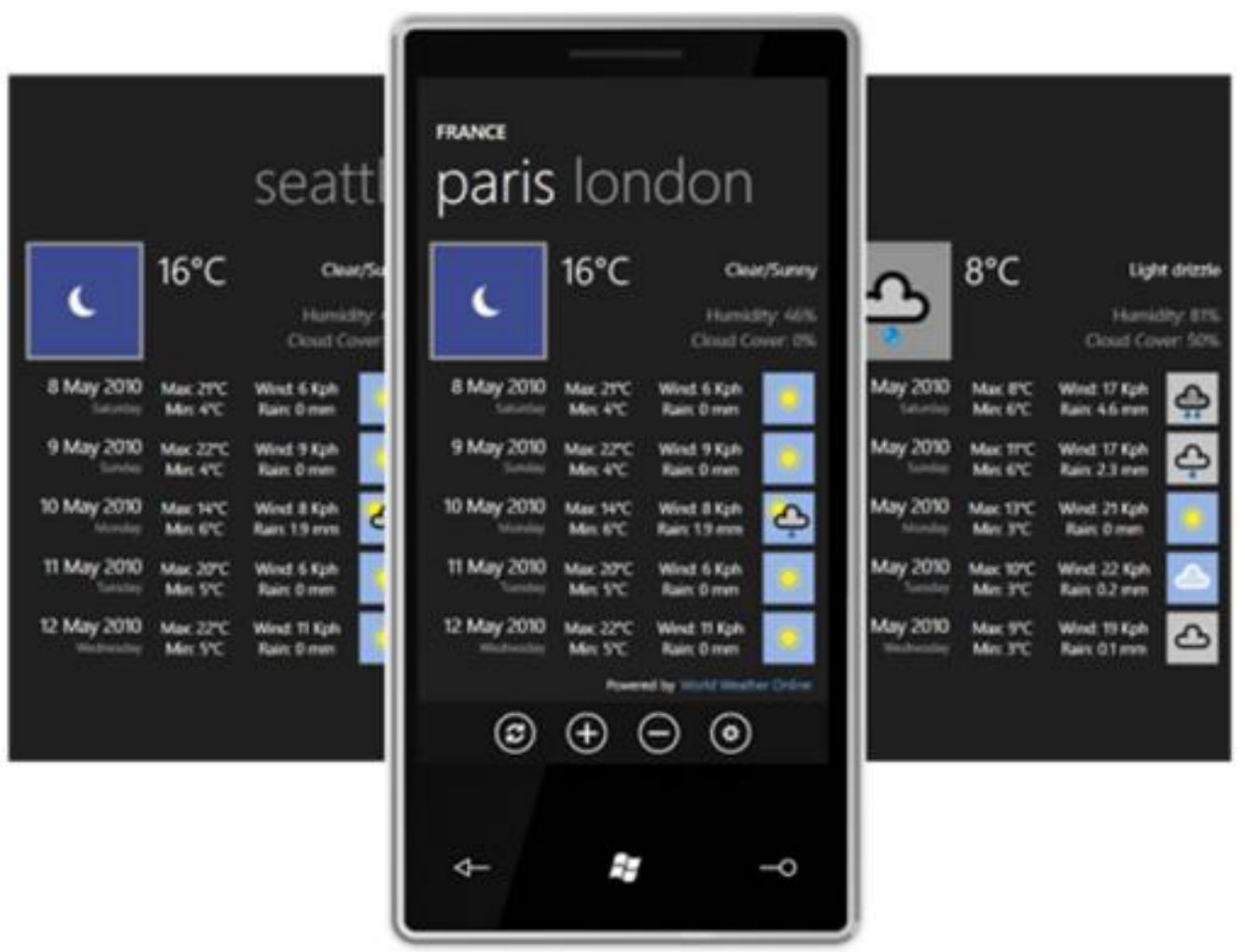

Figure 9: android application 


\section{International Journal of Science and Research (IJSR) \\ ISSN (Online): 2319-7064}

Index Copernicus Value (2013): 6.14 | Impact Factor (2015): 6.391

\section{Comparison of Android and Windows Phone Tools}

Visual Studio 2010 Express for Windows Phone is a fullfeatured Integrated Development Environment (IDE). Visual Studio 2010 facilitates designing, developing, and debugging of Windows Phone applications. Other Windows Phone tools that can help you in the development cycle of the Windows Phone application are:
- Expression Blend

- XNA Game Studio

- Windows Phone Emulator

Compared to Android developer tools, the Windows Phone developer tools offer richer functionality. The following table gives an overview of the functionality of each of these tools. The table also indicates the equivalent tools that you would use for Android application development.

\begin{tabular}{|c|c|c|c|}
\hline Functionality & Audience & Android tools & Windows Phone tools \\
\hline $\begin{array}{l}\text { Primary UI design: Colors, } \\
\text { gradients, and animation }\end{array}$ & UI designers & \multirow{2}{*}{$\begin{array}{c}\text { Defined in XML(No } \\
\text { WYSIWYG) } \\
\text { (or tools like ADT UI } \\
\text { plug-in or DroidDraw) }\end{array}$} & $\begin{array}{c}\text { Pixel accurate WYSIWYG layout using Expression } \\
\text { Blend }\end{array}$ \\
\hline UI design & $\begin{array}{l}\text { UI designers and } \\
\text { programmers }\end{array}$ & & $\begin{array}{c}\text { Visual Studio } 2010 \text { Express and Expression Blend for } \\
\text { Windows Phone }\end{array}$ \\
\hline Application development (coding) & Programmers & Eclipse & Visual Studio 2010 Express for Windows Phone \\
\hline Game development (coding) & Programmers & Eclipse & XNA Game Studio \\
\hline Testing / Emulation & Testers & Android Emulator & $\begin{array}{c}\text { Windows Phone Emulator in Visual Studio } 2010 \\
\text { Express }\end{array}$ \\
\hline
\end{tabular}

As you plan to develop applications for Windows Phone, you can continue to leverage the skillsets in your team. You can use the Android team structure and overall development process to build applications for Windows Phone. The Windows Phone toolset ensures that the entire team of designers, developers, and testers familiar with Android development tools will find it easy to migrate to the Windows Phone toolset.

\section{The Conclusion}

The subject of this paper presents actual points for all researchers because it discusses mobile programming .The paper gives an explanation of windows platform to android application developers to make windows phone a very attractive destination for developers to build applications and games. We explained the developers tools and windows phone architecture to run on multiple phones and let you develop consumer application ,business application ,and games . Compared to android developers tools , the windows phone developers tools offer richer functionality . Android and windows phone are some sort of convertible and they are both made to do the same thing, but their tools are different, if you use android application and windows phone.

\section{References}

[1] Al-KhulaidiAbdualmajed Ahmed Ghaleb , IphonProgramming On Windows Phone 7,Yemen ,J.I.T.N,Vol $2,2014$.

[2] Poslad, Stefan (2009). Ubiquitous Computing Smart Devices, Smart Environments and Smart Interaction. Wiley. ISBN 978-0-470-03560-3

[3] K. Anderson and T. J. Hickey. "SILK - a playful blend of Scheme and Java. In Proceedings of the Scheme and Functional Programming Workshop", September 2000, Amsterdam

[4] Apple Inc." The iPhone development center", 2010. http://developer.apple.com/iphone/.

[5] P. Bothner. Kawa. "Compiling dynamic languages to the Java VM". In Proceedings of the annual conferenceon USENIX Annual Technical Conference,
ATEC '98, pages 41-41, Berkeley, CA, USA, 1998. USENIX Association, London.

[6] Weiser, Mark (1991). "The Computer for the TwentyFirst Century". Scientific American 265 (3): 94-104. doi:10.1038/scientificamerican0991-94.

[7] Beddall-Hill, Nicola, Jabbar, Abdul and Al Shehri, Saleh (2011) "Social Mobile Devices as Tools for Qualitative Research in Education: iPhones and iPads in Ethnography, Interviewing, and Design-Based Research". Journal of the Research Center for Educational Technology, 7 (1). pp. 67-90. ISSN 1948075X.http://eprints.hud.ac.uk/10507/

[8] T. V. Cutsem, S. Mostinckx, E. G. Boix, J. Dedecker, and W. D. Meuter. Ambienttalk: "Object-oriented event-driven programming in mobile ad hoc networks". In SCCC '07: Proceedings of the XXVIInternational Conference of the Chilean Society of Computer Science, pages 3-12, Washington, DC, USA,2007. IEEE Computer Society.

[9] T. V. Cutsem, S. Mostinckx, and W. D. Meuter." Linguistic symbiosis between event loop actors and threads. Computer Languages", Systems \& Structures, 35(1):80-98, 2009.

[10] J. Dedecker, T. V. Cutsem, S. Mostinckx, and W. D. Meuter. "Ambient-oriented programming in AmbientTalk". In Proceedings of the 20th European Conference on Object-oriented Programming (ECOOP,pages 230-254. Springer, 2006.

\section{Author Profile}

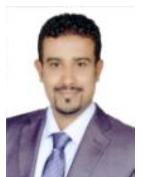

Dr. Abdualmajed Ahmed Ghaleb Al-Khulaidi is Assistant Professor at Software Engineering at Sana'a University, Computer science section, college of Education, Arts and Sciences, Marib Branch, Yemen, His Scientific Interests includes Software Engineering, Distributed Systems, MobileProgramming, Networks,Data Mining ,Database, Artificial Intelligence , Parallel Programming, Data Structures ,Operating Systems, Systems Analysis.

Scientific articles and conferences that I have are 25 ones. 